\title{
Ciprofloxacin-loaded sodium alginate/poly (lactic-co-glycolic acid) electrospun fibrous mats for wound healing
}

\author{
Liu, Xiaoli; Nielsen, Line Hagner; Klodzinska, Sylvia Natalie; Nielsen, Hanne Mørck; Qu, Haiyan; \\ Christensen, Lars Porskjær; Rantanen, Jukka; Yang, Mingshi
}

\author{
Published in: \\ European Journal of Pharmaceutics and Biopharmaceutics
}

Link to article, DOI:

10.1016/j.ejpb.2017.11.004

Publication date:

2017

Document Version

Peer reviewed version

Link back to DTU Orbit

Citation (APA):

Liu, X., Nielsen, L. H., Klodzinska, S. N., Nielsen, H. M., Qu, H., Christensen, L. P., Rantanen, J., \& Yang, M. (2017). Ciprofloxacin-loaded sodium alginate/poly (lactic-co-glycolic acid) electrospun fibrous mats for wound healing. European Journal of Pharmaceutics and Biopharmaceutics, 123, 42-49. https://doi.org/10.1016/j.ejpb.2017.11.004

\section{General rights}

Copyright and moral rights for the publications made accessible in the public portal are retained by the authors and/or other copyright owners and it is a condition of accessing publications that users recognise and abide by the legal requirements associated with these rights.

- Users may download and print one copy of any publication from the public portal for the purpose of private study or research.

- You may not further distribute the material or use it for any profit-making activity or commercial gain

- You may freely distribute the URL identifying the publication in the public portal 


\section{Accepted Manuscript}

Ciprofloxacin-loaded sodium alginate/poly (lactic-co-glycolic acid) electrospun fibrous mats for wound healing

Xiaoli Liu, Line Hagner Nielsen, Sylvia Natalie Klodzinska, Hanne Mørck

Nielsen, Haiyan Qu, Lars Porskjær Christensen, Jukka Rantanen, Mingshi Yang

PII:

S0939-6411(17)30417-4

DOI: https://doi.org/10.1016/j.ejpb.2017.11.004

Reference:

EJPB 12628

Official Journal of
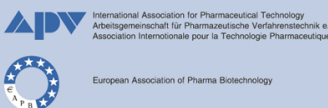

To appear in: $\quad$ European Journal of Pharmaceutics and Biopharmaceutics

Received Date: $\quad 31$ March 2017

Revised Date: $\quad 30$ October 2017

Accepted Date: $\quad 6$ November 2017

Please cite this article as: X. Liu, L.H. Nielsen, S.N. Klodzinska, H.M. Nielsen, H. Qu, L.P. Christensen, J. Rantanen, M. Yang, Ciprofloxacin-loaded sodium alginate/poly (lactic-co-glycolic acid) electrospun fibrous mats for wound healing, European Journal of Pharmaceutics and Biopharmaceutics (2017), doi: https://doi.org/10.1016/j.ejpb. 2017.11.004

This is a PDF file of an unedited manuscript that has been accepted for publication. As a service to our customers we are providing this early version of the manuscript. The manuscript will undergo copyediting, typesetting, and review of the resulting proof before it is published in its final form. Please note that during the production process errors may be discovered which could affect the content, and all legal disclaimers that apply to the journal pertain. 


\section{Ciprofloxacin-loaded sodium alginate/poly (lactic-co-glycolic acid) electrospun fibrous mats for wound healing}

Xiaoli Liu ${ }^{1}$, Line Hagner Nielsen ${ }^{2}$, Sylvia Natalie Klodzinska ${ }^{1}$, Hanne Mørck Nielsen ${ }^{1}$, Haiyan $\mathrm{Qu}^{3}$, Lars Porskjær Christensen ${ }^{3}$, Jukka Rantanen ${ }^{1}$, Mingshi Yang ${ }^{1,4, A}$

${ }^{1}$ Department of Pharmacy, University of Copenhagen, Universitetsparken 2, DK-2100 Copenhagen, Denmark; ${ }^{2}$ Department of Micro- and Nanotechnology, Technical University of Denmark, Ørsteds Plads, Building 345C, DK-2800 Kongens Lyngby, Denmark; ${ }^{3}$ Department of Chemical Engineering, Biotechnology and Environmental Technology, University of Southern Denmark, Campusvej 55, DK-5230 Odense M, Denmark; ${ }^{4}$ Wuya College of Innovation, Shenyang Pharmaceutical University, Wenhua Road No. 103, 110016 Shenyang, China

${ }^{A}$ Corresponding author: Mingshi Yang(mingshi.yang@sund.ku.dk) 


\begin{abstract}
Wound dressings should ideally be able to maintain high humidity, remove excess wound exudate, permit thermal insulation, provide certain mechanical strength, and in some cases deliver antibiotics to prevent infections. Until now, none of the existing wound dressing products can meet all these requirements. To design a wound dressing with as many of the aforementioned features as possible, in this study, we attempted to prepare ciprofloxacin (CIP), an antibiotic, loaded electrospun hydrophobic poly (lactic-co-glycolic acid) (PLGA) fibrous mats modified with hydrophilic sodium alginate (ALG) microparticles. The results showed that ALG could improve the wettability, water absorption capacity, and enhance the release rate of ciprofloxacin from the PLGA fibrous mats. In addition, the addition of ALG reduced the stiffness of PLGA fibrous mats for better protection of the injured area as indicated by the Young's Modulus. Moreover, the burst release of CIP resulted from the addition of ALG seemed to provide an improved antibacterial effect to the PLGA mats . This study demonstrated the potential of combining hydrophilic and hydrophobic polymers to design the desired wound dressings via the electrospinning process.
\end{abstract}

Key words: electrospinning; hydrodynamic methods; hydrophilicity; mechanical properties; antimicrobial activity; water-solid interactions 


\section{Introduction}

Wound healing is a complex process beginning with haemostasis, inflammation, and removal of damaged matrix components, followed by tissue formation and remodeling[1]. Generally, inflammation occurs immediately after tissue damage, and new tissue formation occurs 2-10 days after injury with cellular proliferation and migration of different cell types to the site of injury. Remodeling is the final stage of wound healing, beginning 2-3 weeks after injury and continuing for a year or more. Healing of chronic wounds normally takes more than 12 weeks and often the wounds reoccur[2]. Chronic wounds are often heavily contaminated and usually involye significant tissue loss affecting vital structures of bones, joints, and nerves. Such wounds fail to heal due to repeated trauma to the injured area, and moreover physiological conditions such as diabetes, persistent infections, and poor primary treatment can also affect the healing[3]. Furthermore, impaired wound healing can lead to an excessive production of exudates causing maceration of the healthy skin tissue around the wound[4].

For better wound healing, a non-toxic and non-adherent wound dressing should be utilized. It should perform as a protective barrier, but also aid healing of the wound by fulfilling an array of requirements such as maintaining high humidity, removing excess wound exudates, and permitting thermal insulation. Further, it is beneficial if the dressing can allow for gas exchange, conforming to the wound surface and also be impermeable to bacteria[5-7]. However, none of the currently used wound dressing can fulfil all these reqirements. The majority of applied wound dressings can be classified as hydrocolloids, hydrogels, foams, or films $[6,8,9]$. Hydrocolloids and hydrogels can maintain moist wound environments and are usually used together with other functional layers. However, for the hydrocolloid wound dressings, the excessively moist environment caused by absorbing wound fluids might accelerate autolysis of necrotic tissue and increase the risk of infection[10]. In contrast, the hydrogels can swell or shrink in a reversible way, depending on the $\mathrm{pH}$ and ionic strength of the aqueous environment. This kind of wound dressing is more efficient when used for wounds with few exudates[8]. Foam-type dressings are also used for moderate/high draining wounds due to their high absorbance capacity, whereas films are normally durable, semipermeable, and impermeable to liquid and bacterial contamination[11]. However, medicated wound dressings based on films, which are characterized by incorporating an active agent in the films, do not have the capacity to absorb exudates[12]. To incorporate as many of the above-mentioned requirements as possible, new design of wound dressings are therefore desperately needed, and ideally they should be produced with an easy operating process.

Nanofibrous mats produced by electrospinning have been reported to be good candidates for wound healing due to their unique properties, e.g. mimicking the biostructure of the extracellular matrix with high porosity aiding in exchanging of liquid and gases with the environment[13-15]. Electrospun fibrous mats (EFMs) possess small pores, which can protect the wound from bacterial penetration via aerosol particle capturing mechanisms[16]. In addition, therapeutic agents such as antimicrobials can be incorporated into the EFMs and thereby provide protection of the wound from contamination. 
Numerous natural polymers can be electrospun into fibrous mats for wound dressings, they are in general biocompatible and biodegradable, and moreover, have great similarity to the extracellular matrix. In previous studies, materials such as silk[17] and collagen[18] have been elecrospun into nanofibrous mats for wound healing. However, natural polymers exhibit a large variation in physicochemical characteristics due to the fact that they are materials derived from animals and plants from different sources and forms [19], and in addition some natural polymers cannot be electrospun into fibers owing to a few reasons such as high viscosity associated with their high molecular weight, degradation in solution, and difficulty to dissolve in adequate solvents[20, 21]. Furthermore, nanofibers prepared from most natural polymers lack the desired mechanical properties with regards to less than $10 \%$ elongation at break[22]. In contrast to natural polymers, synthetic polymers have more diverse physicochemical properties, including some being hydrophilic and others hydrophobic, good mechanical properties, and they are often also cheap, show little batch to batch variations and a higher degree of purity[21, 23]. The disadvantage of synthetic polymers is that they lack the biochemical signatures expressed in native fibers of the body. In order to form suitable and biomimetic nanofibers, natural and synthetic polymers may be combined.

In this study, poly (lactic-co-glycolic acid) (PLGA) and sodium alginate (ALG) were combined and processed by using electrospinning to obtain nanofibrous mats with desired physicochemical properties for wound healing. ALG is one of the most studied and applied polysaccharides in wound healing, due to not only its abundance in nature, and its high water uptake potential[24] and PLGA was chosen because it is a biocompatible and biodegradable synthetic polymer, approved by FDA for a wide pharmaceutical application. Ciprofloxacin (CIP) was incorporated into the nanofibrous mats as a model antibiotic, and the resulting EFMs were characterized in vitro for their physicochemical properties and their antimicrobial activity.

\section{Materials and Methods}

\subsection{Materials}

PLGA (lactic acid (LA): glycolic acid (GA), 75:25, molar ratio) with inherent viscosity $\left(25^{\circ} \mathrm{C}, 0.1 \%\right.$ chloroform) in the range 0.8-1.2 dl/g (RG750, MW 120,000-190,000 g/mol) was purchased from Evonik industries (Darmstadt, Germany). Trifluoroethanol, Mueller-Hinton broth, tryptic soy agar and chloroform were obtained from Sigma-Aldrich (Brøndby, Denmark) together with ciprofloxacin (CIP), sodium alginate (ALG) and phosphate buffered saline (PBS) tablets. UltraCruz $^{\circledR}$ Petri Dishes sc-351865, $100 \mathrm{~mm} \times 15 \mathrm{~mm}$ were purchased from Santa Cruz Biotechnology (Santa Cruz, CA, USA), and Staphylococcus aureus-15981 WT was kindly provided by the Institute of Immunology and Microbiology, University of Copenhagen, Denmark. Deionised water was obtained from an SG Ultra Clear water system (SG Water USA, Nashua, NH, USA) and was freshly produced in all cases.

\subsection{ALG particles preparation by spray drying}

ALG particles were prepared using a Büchi B-290 mini spray dryer (Buchi Labortechnik, Falvil, Switzerland) equipped with an inert loop B-295 (Buchi Labortechnik). $0.5 \%$ (w/v) of ALG solution 
in water was prepared by spray drying at $170{ }^{\circ} \mathrm{C}$ of inlet temperature resulting in an outlet temperature of approximately $90{ }^{\circ} \mathrm{C}$. The drying air flow rate was kept at $35 \mathrm{~m}^{3} / \mathrm{h}$ with an atomizing air flow rate of $667 \mathrm{~L} / \mathrm{h}$ and feed flow rate of $2 \mathrm{~mL} / \mathrm{min}$.

\subsection{Preparation of electrospun fibrous mats (EFMs)}

$25 \%(\mathrm{w} / \mathrm{v})$ of PLGA was dissolved in a binary mixture of trifluoroethanol and chloroform (4:1 v/v), and left overnight to dissolve. Electrospinning was conducted at room temperature using a syringe pump (Harvard Apparatus, Quebec, Canada) and a 16 gauge needle (inner diameter $1.19 \mathrm{~mm}$ and outer diameter $1.65 \mathrm{~mm}$ ) in a high-voltage supply (20 kV, max.) (Glassman High Voltage, High Bridge, NJ, USA). The flow rate was $10 \mu \mathrm{L} / \mathrm{min}$, and the distance between the nozzle tip to the grounded collector was $10 \mathrm{~cm}$. The fibers were collected on a stationary plate, and the voltage was adjusted to get a stable cone-jet with minimum change. In order to get homogenous mats for tensile strength test, a rotating drum $(1500 \mathrm{rpm})$ was used to collect the electrospun samples.

For preparation of CIP-loaded EFMs, CIP was dissolved in trifluoroethanol, while ALG particles were re-suspended in chloroform. Subsequently, the solution and suspension were added to the PLGA solution (prepared as described above). The drug loading was approximately $1 \%(\mathrm{w} / \mathrm{w})$ relative to the PLGA mass ratio, and the amount of ALG in the EFMs was varied to be either 1:100, 2.5:100, and 4:100 (ALG:PLGA, w/w).

\subsection{Morphology of the EFMs}

The morphology of the EFMs was characterized using scanning electron microscope (SEM, Hitachi High-Tech HITACHI, Tokyo, Japan). The EFMs were mounted on metal stubs using double-sided adhesive tape and the samples were coated under vacuum with gold in an argon atmosphere prior to examination followed by imaging at an accelerating voltage of $5 \mathrm{kV}$. The diameter of electrospun fibers was determined by measuring the geometry of around 100 individual fibers from the SEM images using the instrument software (TM3030).

\subsection{Water contact angle}

For evaluation of the influence of ALG on the hydrophilicity of the PLGA EFMs, the water contact angle was measured using a drop shape analyzer (Mode: DSA100, KRÜSS, Hamburg, Germany). One droplet of water $(20 \mu \mathrm{L})$ was added on the EFMs, and images of the water droplet were acquired at $0 \mathrm{~min}$, after $30 \mathrm{~min}$ and after $2 \mathrm{~h}$ to observe the change of water contact angle over time. Each specimen was tested in triplicate.

\subsection{Water uptake - swelling of the EFMs}

The PLGA and ALG/PLGA EFMs were cut into $50 \mathrm{~mm} \times 40 \mathrm{~mm}$ squares for assessment of the swelling properties of the fibers. The study was performed as a PBS absorption study. In brief; preweighed EFMs (approximately $50 \mathrm{mg}$ ) were placed in $50 \mathrm{~mL}$ centrifuge tubes containing $40 \mathrm{~mL}$ of PBS at $\mathrm{pH} 7.4$, and incubated at $37.0 \pm 0.1{ }^{\circ} \mathrm{C}$ for $4 \mathrm{~h}$. All the samples were tested in triplicate. The wet weight of the samples was determined by weighing immediately after dehydrating the samples with filter paper to absorb water present at the fiber film surface after removal from PBS. The water uptake of the EFMs in PBS was then calculated using Eq. 1 and refered to as swelling (\%): 


$$
\text { Swelling }(\%)=\left(\left(\mathrm{w}_{1}-\mathrm{w}_{0}\right) / \mathrm{w}_{0}\right) \times 100 \%
$$

where $\mathrm{w}_{0}$ and $\mathrm{w}_{1}$ are the weights of the EFMs before and after immersing in the PBS, respectively.

\subsection{Water sorption/desorption}

Water sorption/desorption studies of the EFMs were performed using a VTI Vapor Sorption Analyzer (SGA-100, VTI Corporation, Hialeah, FL, USA). The water sorption/ desorption profile was measured at $25^{\circ} \mathrm{C}$ and $60{ }^{\circ} \mathrm{C}$. The EFMs were dried at $60{ }^{\circ} \mathrm{C}$ and close to $0 \%$ relative humidity $(\mathrm{RH})$, weighed and then weighed after increasing the $\mathrm{RH}$ in $10 \%$ steps from approximately $0 \%$ to $90 \% \mathrm{RH}$ at $25^{\circ} \mathrm{C}$. Equilibrium was assumed when the weight change was $<0.01 \%$ over a period of $7 \mathrm{~min}$. Each specimen was measured in triplicate.

\subsection{Tensile strength}

The tensile strength measurements were carried out utilizing a TA.XT plus texture analyzer (Stable Micro Systems, Godalming, UK) using the method described in a previous study[25]. The EFMs were cut into quadrangles of $5 \mathrm{~cm} \times 1 \mathrm{~cm}$, and the thickness of the mats was measured by an electronic micrometer (Schut Geometrical Metrology, Groningen, Netherland). In order to avoid breakage of the sample during the sample fixing, the EFMs were inserted into the gripping part of the probe together with aluminum foil (cut out before the measurements). The test speed was 0.04 $\mathrm{mm} / \mathrm{s}$ and the gripping distance was $40 \mathrm{~mm}$. Each specimen was tested five times.

\subsection{Drug release}

The release behavior of CIP out of the EFMs was studied in PBS at pH 7.4. EFMs were cut into specific shape $(5 \mathrm{~cm} \times 4 \mathrm{~cm})$, and placed in a centrifuge tube containing $10 \mathrm{~mL}$ of PBS. The tubes were shaking in a water bath at $100 \mathrm{rpm}$ at $37{ }^{\circ} \mathrm{C}$, and at designated time intervals $(1,2,4,8,12$, and $24 \mathrm{~h}$ followed by $2,3,4,5,6,7$, and 10 days, then afterwards every week until 14 weeks). $1 \mathrm{~mL}$ release medium was taken out and $1 \mathrm{~mL}$ fresh PBS was immediately added to maintain the volume. Each specimen was tested in triplicates, and the CIP concentration was measured by UV spectrophotometer (Evolution 300, Thermo Fisher Scientific, Waltham, MA, USA) at $260 \mathrm{~nm}$.

\subsection{Biodegradation behavior}

The biodegradation behavior of the EFMs were evaluated by measuring the weight change after incubating in PBS for a period of time. The initial mass of the samples were measured, and the samples were then degraded by placing them in PBS, pH 7.4 at $37^{\circ} \mathrm{C}$. At selected time intervals $(1$, $3,5,7,9,11,13$, and 15 weeks), the EFMs were removed from the solution, and the water on the surface was adsorbed by filter paper followed by drying the EFMs in a vacuum oven for $24 \mathrm{~h}$, and then weighed. Each specimen was tested in triplicate. The biodegradation percentage, D (\%), was defined as in Eq. 2:

$$
\mathrm{D}(\%)=\left(\mathrm{w}_{\mathrm{i}}-\mathrm{w}_{\mathrm{t}} / \mathrm{w}_{\mathrm{i}}\right) \times 100 \%
$$

where $\mathrm{w}_{\mathrm{i}}$ is the initial weight of the sample and $\mathrm{w}_{\mathrm{t}}$ is the dried weight of the sample at the selected time intervals. 


\subsection{Antimicrobial activity on solid culture medium}

Tryptic Soy Agar (TSA) was prepared by dissolving $40 \mathrm{~g}$ of dehydrated TSA medium in $1 \mathrm{~L}$ of purified, filtered water and sterilized at $121{ }^{\circ} \mathrm{C}$ for $15 \mathrm{~min}$. Each agar plate was prepared by pouring $10 \mathrm{~mL}$ of molten medium into a Petri dish $(100 \mathrm{~mm} \times 15 \mathrm{~mm})$, and then the plate was allowed to solidify for 1 hour under sterile conditions in the laminar flow. Prior to use, bacteria from cryostock were grown overnight in Mueller-Hinton broth (MHB) at $37{ }^{\circ} \mathrm{C}$. The overnight inoculum was transferred to fresh MHB and incubated for 2-3 h to reach exponential growth phase. Bacteria suspensions were adjusted to $0.5 \mathrm{McF}$ arland suspension $\left(1 \times 10^{8} \mathrm{CFU} / \mathrm{mL}\right.$, optical density at 600 $\mathrm{nm}(\mathrm{OD} 600)=0.08-0.1)$ and further diluted 1:20 in MHB. A sterile inoculation loop was used to transfer bacteria from suspension to agar plates for contamination check every time bacteria were grown from cryostocks.

For plating bacteria on the agar plates, $10 \mu \mathrm{L}$ of adjusted bacteria suspension was added to $100 \mu \mathrm{L}$ of MHB to yield 2-5 $\times 10^{5} \mathrm{CFU} / \mathrm{mL}$. $100 \mu \mathrm{L}$ of the suspension was then swabbed uniformly across the solid culture, then a round EFM (diameter, $10 \mathrm{~mm}$ ) was placed on the surface of solid culture medium. Subsequently, the solid culture plates were cultivated in an INCUCELL incubator (MMM Medcenter Einrichtungen, München, Germany) at $37 \pm 2{ }^{\circ} \mathrm{C}$ for $20 \mathrm{~h}$. The relative size of the inhibition zone was measured with calipers and recorded. All the samples were tested in triplicate. Broth micro dilution was used to determine the minimal inhibitory concentration (MIC) for S.aureus $15981 \mathrm{WT}$ and CIP. Twofold dilutions of CIP in MHB (in the range $128-0.06 \mu \mathrm{g} / \mathrm{ml}$ ) were prepared and $100 \mu \mathrm{L}$ of each concentration was pipetted into a separate well of a sterile polystyrene flat-bottom 96-well plate (Costar Corning® 3596 cell culture plates, Corning, NY, USA). $10 \mu \mathrm{L}$ of adjusted bacteria suspension was then added to yield $5 \times 10^{4} \mathrm{CFU} /$ well. For growth control, an antibiotic solution was replaced with MHB. $100 \mu \mathrm{L}$ of MHB without bacteria was used as control of sterility. The broth dilution plates were incubated as described above. Visual turbidity or sedimentation was defined as bacterial growth, whereas lack of turbidity was considered as inhibition of growth. The lowest concentration of antibiotic that inhibited visual turbidity was defined as MIC value.

\subsection{Statistics}

The data are represented as means \pm standard deviation (SD). Statistics were carried out using Origin software (v9.1, academic, OriginLab, Northampton, MA, USA). P-values below 5\% (p < 0.05 ) were considered statistically significant, unless otherwise stated.

\section{Results and discussion}

To accommodate both hydrophilic ALG and hydrophobic PLGA in the same EFM, ALG was first spray-dried into particles, and then suspended in PLGA solution. Subsequently, this suspension was processed using the elestrospinning process. It has been shown that co-axial electrospinning could be used to process two polymer solutions with different hydrophilicity properties into nanofibrous mats[26, 27]. However, maintaining the stability of the cone-jet in the co-axial electrospinning 
process is still a challenge due to distinct properties of the two polymer solutions, which may result in non-uniform fiber structure and defects in the mats[14, 28]. Another challenge is obtaining uniform distribution of the active drug compounds incorporated into the fibers. The drug molecules may migrate from one polymer to the other polymer matrix in the electropinning process[29], and the charge repulsion may lead to an enrichment of charged or polarizable agents on the surface of the fibers[30]. In contrast, electrospinning of heterogeneous systems such as suspension or emulsion using a single nozzle has been proven to be successful in terms of the stability of the electrospinning process and the formation of uniform fibers[31, 32]. In this study, a stable cone-jet of the ALG-PLGA suspension was obtained and the physicochemical properties of the resulting nanofibrous mats were characterized as described in the following sections[33].

\subsection{SEM of the EFMs}

Spray dried ALG particles were spherical with a wide size distribution ranging from approximately $100 \mathrm{~nm}$ to $15 \mu \mathrm{m}$ (Fig. 1A). The particles were readily suspended in PLGA-trifluoroethanol solution prior to electrospinning. The EFMs exhibited a uniform diameter except that some beaded fibers were formed in the formulations with a relatively larger amount of spray-dried ALG particles (Fig. 1B-F). It can be explained by the fact that the size of some of the ALG particles was bigger than the diameter of the electrospun fibers. Nevertheless, stable cone-jets were observed at a voltage of approximately $9 \mathrm{kV}$ for all the processings. The diameter of the resulting EFMs is showed in Table 1. The results showed that the diameter of EFMs was significantly decreased $(p<0.05)$ with the modification of ALG particles compared to pure PLGA EFM. With addition of CIP, there was no significance change between pure PLGA EFM and CIP modified ALG/PLGA EFMs.

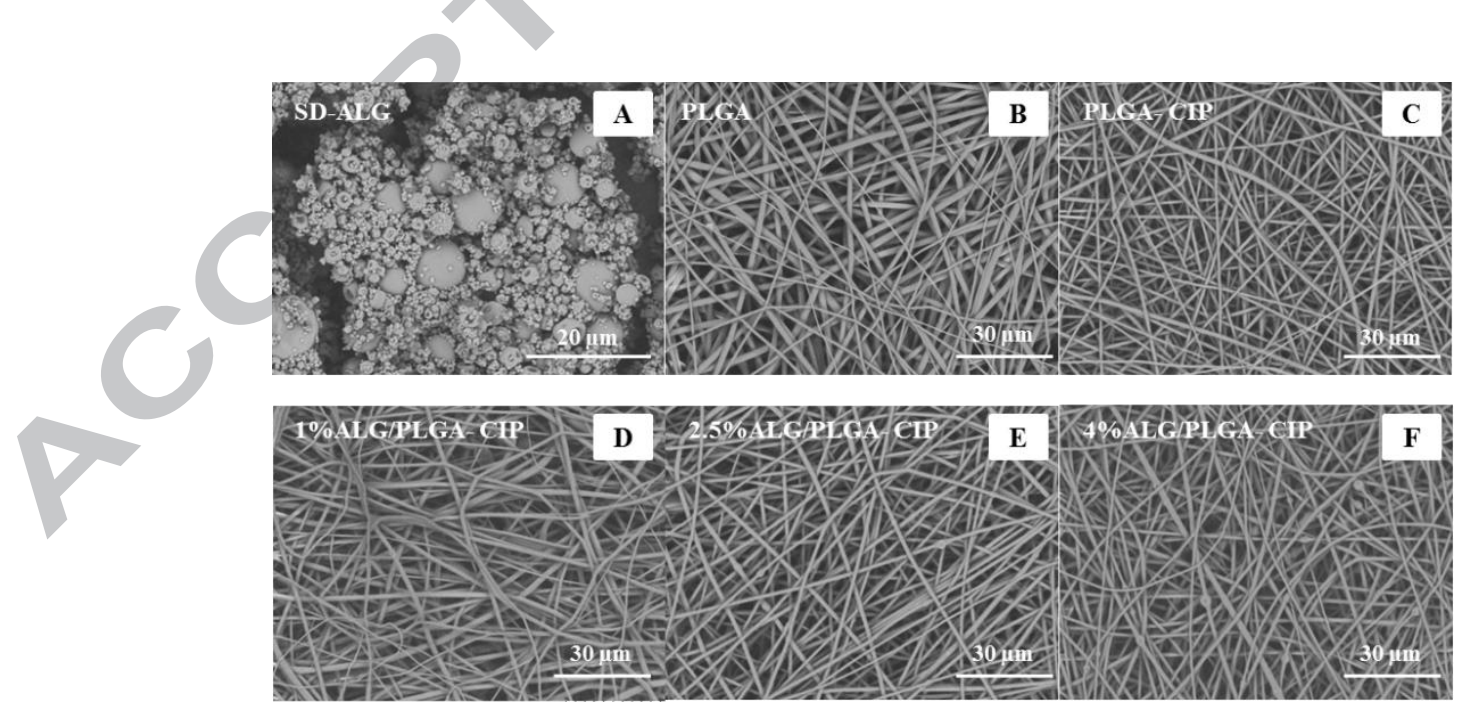

Fig. 1 SEM images of the spray dried ALG particles and EFMs with and without ALG particles. A: spray dried ALG particles; B: PLGA EFM; C: PLGA-CIP EFM; D: 1\% ALG/PLGA-CIP EFM; E: 2.5\% ALG/PLGA-CIP EFM; F: 4\% ALG/PLGA-CIP EFM. 
Table 1. The diameter of EFMs with and without ALG particles.

\begin{tabular}{cccc}
\hline \multicolumn{2}{c}{ ALG/PLGA EFM } & \multicolumn{2}{c}{ ALG/PLGA-CIP EFM } \\
\hline $\begin{array}{c}\text { ALG } \\
(\mathrm{w} / \mathrm{w}, \%)\end{array}$ & $\begin{array}{c}\text { Diameter } \\
(\text { mean } \pm \text { SD }, \mathrm{nm})\end{array}$ & $\begin{array}{c}\text { ALG } \\
(\mathrm{w} / \mathrm{w}, \%)\end{array}$ & $\begin{array}{c}\text { Diameter } \\
(\text { mean } \pm \mathrm{SD}, \mathrm{nm})\end{array}$ \\
\hline 0 & $777 \pm 249$ & 0 & $821 \pm 376$ \\
1 & $673 \pm 243^{*}$ & 1 & $877 \pm 431$ \\
2.5 & $633 \pm 156^{*}$ & 2.5 & $749 \pm 316$ \\
4 & $676 \pm 242^{*}$ & 4 & $747 \pm 233$ \\
\hline
\end{tabular}

Note: $* p<0.05$ compared with pure PLGA EFM

\subsection{Water contact angle of the EFMs}

Hydrophilicity is an important quality attribute for wound dressing as it influence cell viability and proliferation rate[34]. To evaluate the hydrophilicity of EFMs, water contact angles of the mats were measured. As expected, due to the hydrophilicity of ALG (water contact angle of 59 ${ }^{\circ}$ [26], loading of ALG particles in the PLGA EFMs lowered the water contact angle as compared to the pure PLGA EFM, especially after 30 and $120 \mathrm{~min}$ (Fig. 2). It was found that from $0 \%$ ALG to 1, 2.5 , and $4 \%$ ALG, the water contact angle decreased from $111.7 \pm 3.0^{\circ}$ to $109.1 \pm 2.6^{\circ}$ to $103.6 \pm$ $1.1^{\circ}$, and to $97.3 \pm 0.7^{\circ}$, respectively after $30 \mathrm{~min}$. After $120 \mathrm{~min}$, the water contact angles of these samples all were decreased to a value lower than $66^{\circ}$, close to the water contact angle of ALG. This observation indicates that ALG particles embedded in the PLGA EFMs can get wet by exposing the mats with water for longer time period. 
PLGA
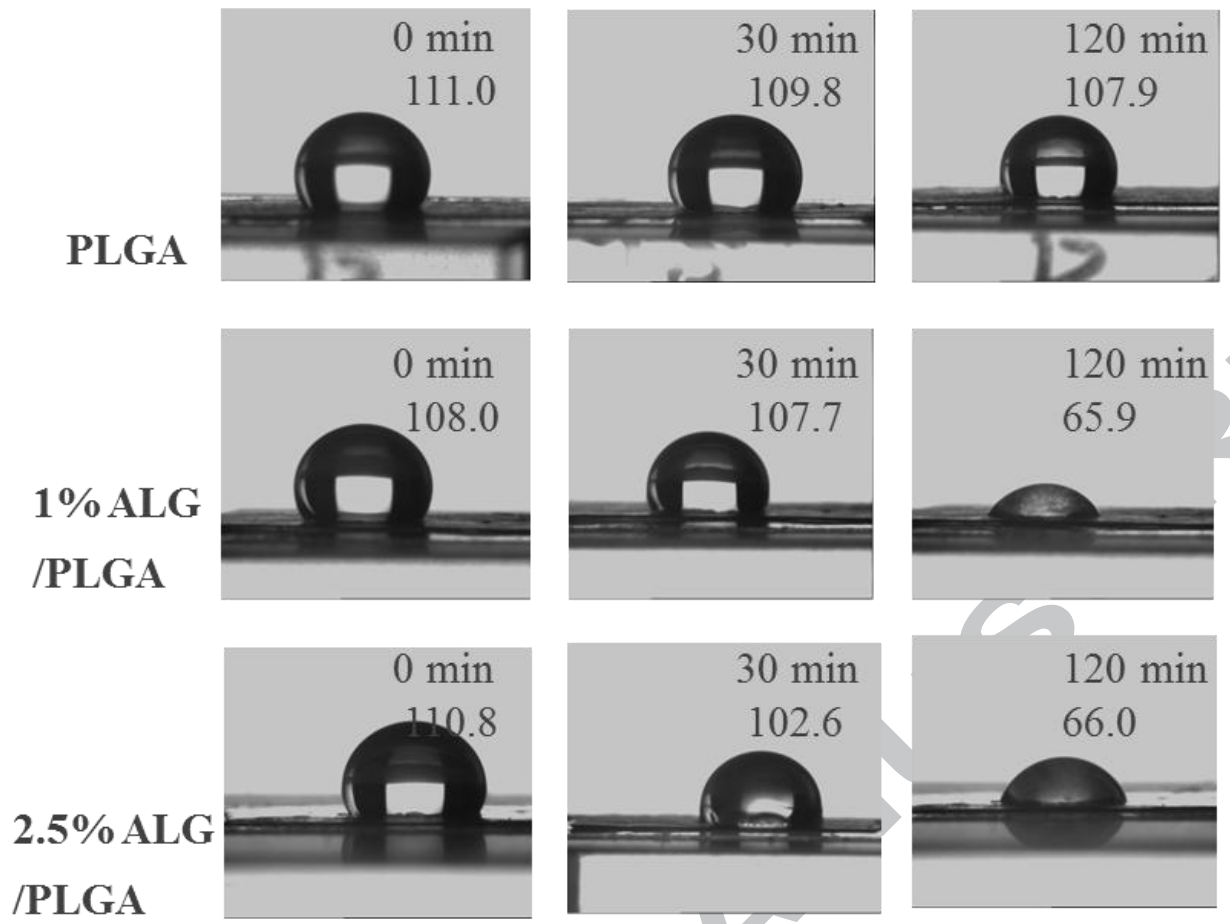

/PLGA
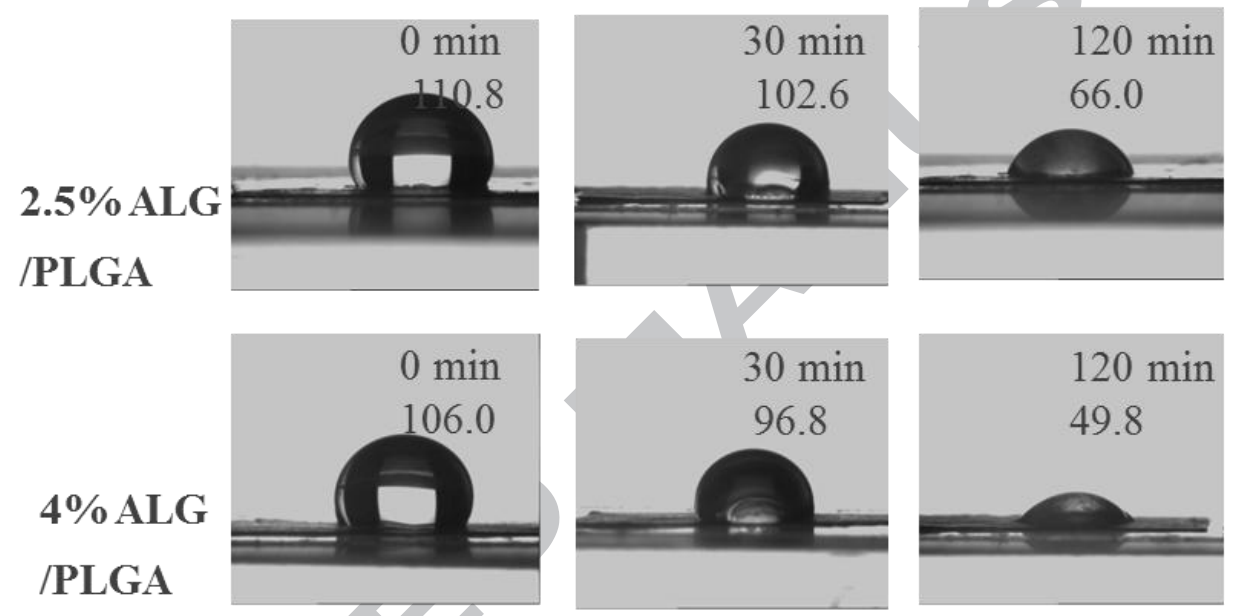

Fig.2 Water contact angle of EFMs without ALG particles, and with 1, 2.5 or 4\% ALG particles. The contact angles were measured at 0,30 and $120 \mathrm{~min}$ in triplicates, and the data shown are representative for the triplicates.

\subsection{Water uptake-swelling of the EFMs}

Water uptake is another critical quality attribute for a wound dressing as it reflects the dressing's capacity to absorb drainage from open wounds. The presence of the hydrophilic polymer ALG obviously enhanced the absorption property to the mats. When incorporating ALG particles into the electrospun fibers, the EFMs absorbed 1.2 to 2.3 times more water than the PLGA EFM (Fig. 3). Especially the EFM loaded with 4\% ALG particles showed a significant improvement of the water uptake capacity compared to the PLGA EFM $(\mathrm{p}=0.012)$. The swelling capacity of the mats in this study was much lower than that of the pure ALG wound dressing (swelling capacity, $1300 \%$ ) due to the limited amount of ALG used to modify the mats[35]. However, the ALG-modified PLGA EFMs reached the same swelling capacity as the one that was prepared from a hydrophilic polymer poly(vinyl alcohol), with the modification of ALG in the same amount[36]. 


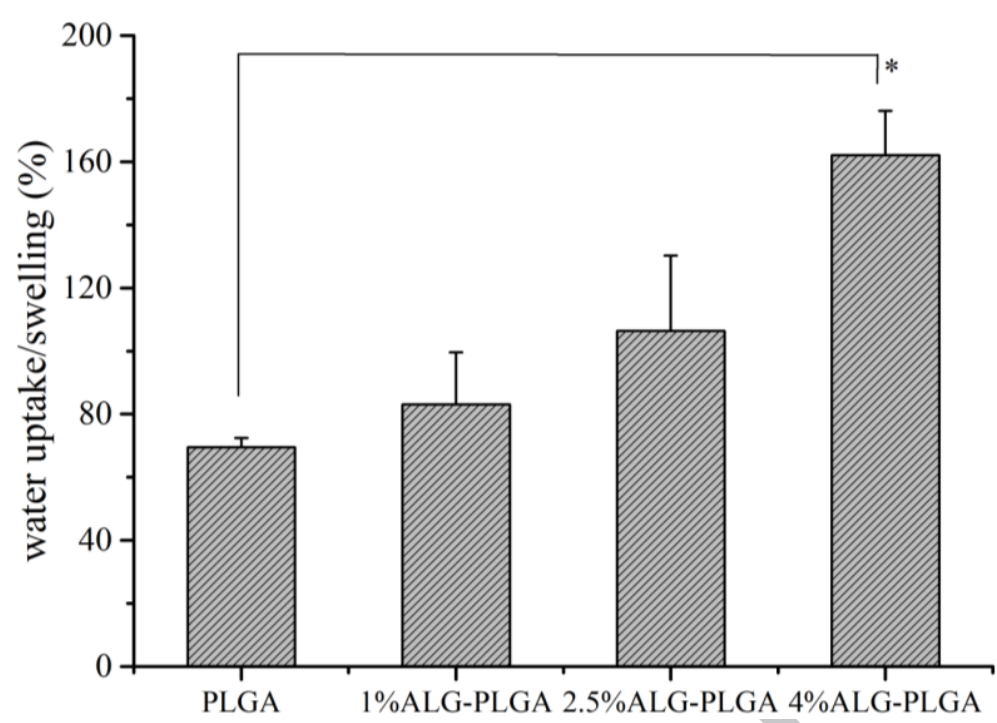

Fig. 3 Swelling capacity of the EFMs with various ALG content ratios when immersed in PBS at pH $7.4($ mean $\pm S D, n=3)$., the * indicate significant difference for $\mathrm{p}<0.05$.

\subsection{Water sorption/desorption}

Beside the swelling ability of EFMs in PBS solution as aforementioned, the water-solid interactions of EFMs were analyzed by the water sorption/desorption analysis. The percentage of water vapor uptake with respect to the dry polymer weight at different $\mathrm{RH}$ is presented in Fig. 4. The EFMs with high amount of ALG particles showed higher water sorbing capacity as compared to the ones modified with relative lower amount of ALG, whereas the effect was negligible when the amount of ALG particles was $1 \%$ as compared to the one without modification of ALG.

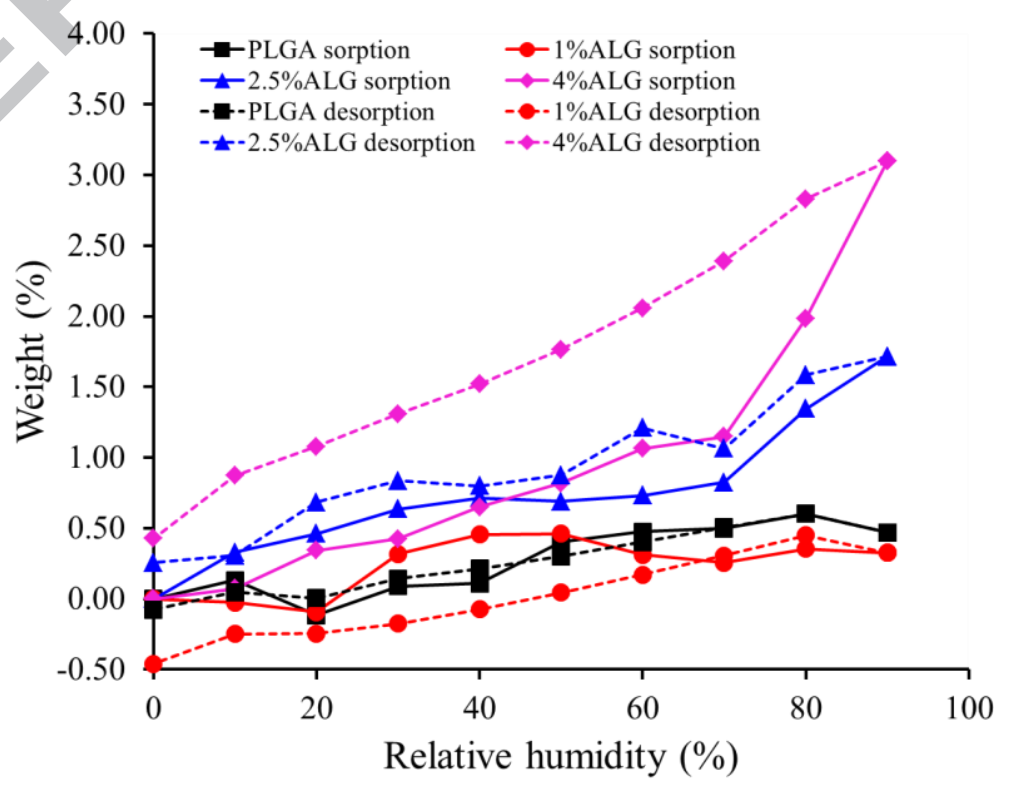


Fig.4 The water sorption/desorption profiles for EFMs without ALG particles, and with 1, 2.5 or $4 \%(\mathrm{w} / \mathrm{w})$ ALG particles. The solid line and dotted line represent sorption and desorption process, respectively, and the data shown are representative for the triplicates.

\subsection{Drug release}

The release profiles of CIP from PLGA EFM and from ALG/PLGA EFMs were obtained in PBS at pH 7.4 (Fig. 6). For all the samples, a burst release can be observed in the first 7 days followed by a plateau stage with slow release of CIP continuing for up to 40 - 50 days. After the slow release phase and until the end of the study (between ca. 50 - 98 days), there was a fast release until a complete release of CIP from the EFMs. Wound healing is a lengthy process and normally takes between one month and several years, and an antimicrobial effect is necessary during the whole process, especially for the first 5 days after the injury[8]. The burst release can be attributed to the fast release of the CIP precipitated on the surface upon drying. The burst release appears increasing with an increase in ALG particles in the fibers, which may be attributed to improved hydrophilicity of the mats upon adding ALG and dissolution of ALG which generated larger surface area for drug diffusion. PLGA is a biodegradable polymer, which can undergo degradation (a decrease in average molecular weight) and subsequently erosion (a decrease in total mass) during the release study. When the polymer began to erode, more water could penetrate deep into the mats. Therefore, CIP was released faster, where both diffusion and polymer erosion contribute significantly to the drug release[37]. To further investigate the release mechanism of the mats, the degradation rate of PLGA was measured and is described in the next section.

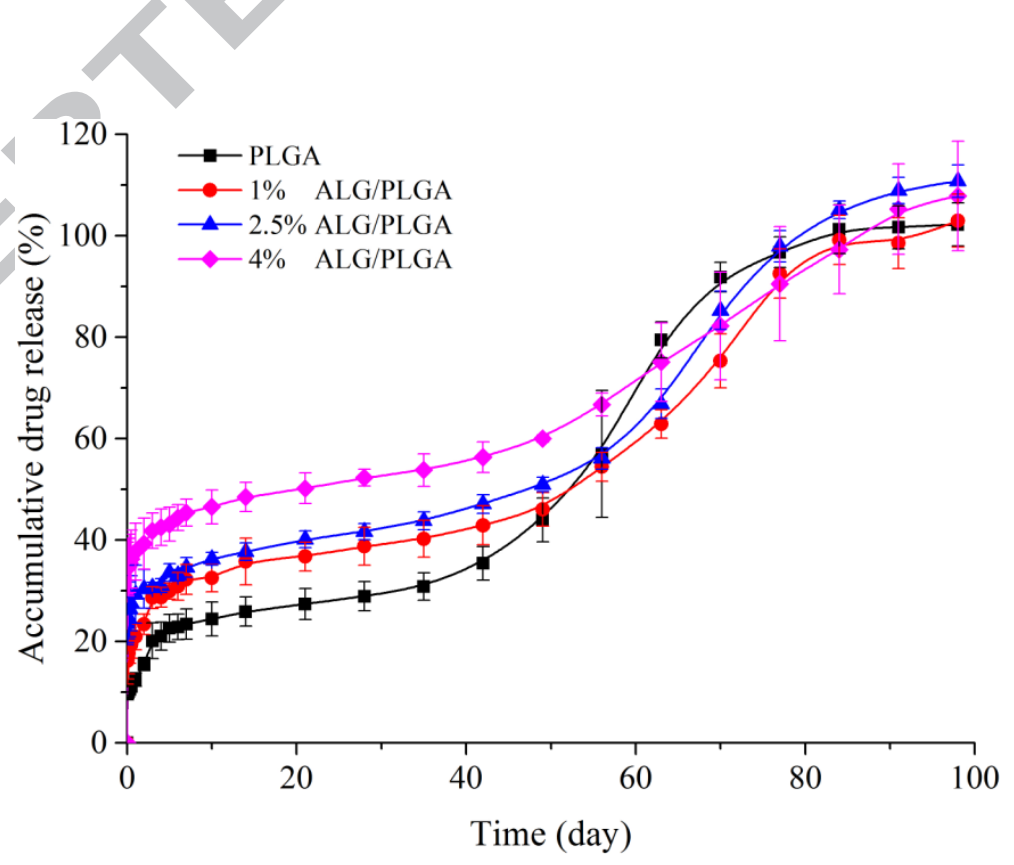

Fig.5 Cumulative release of CIP from PLGA or ALG/PLGA EFMs (containing 1, 2.5 or $4 \%$ $\mathrm{ALG}$ ) in PBS, $\mathrm{pH} 7.4$ at $37^{\circ} \mathrm{C}$ over a period of 98 days (mean $\pm \mathrm{SD}, \mathrm{n}=3$ ). 


\subsection{Biodegradation rate of PLGA}

The biodegradation rate of polymer is important for a drug delivery system and is strongly related to the drug release profile. PLGA is known to degrade upon hydrolysis. The percentage of weight loss of PLGA and ALG/PLGA EFMs was studied and the results shown in Fig. 6. For all EFMs, the weight loss increased over time, but was slow in the first 50 - 80 days. The result was similar to that found in a previous study, where the PLGA with higher molecular weight did not show any detectable change in the degradation until 53 days[38]. After the slow degradation phase, the $4 \%$ ALG/PLGA EFM began to have a fast weight loss from day 50, while the other EFMs started to degrade faster after 80 days. Generally, the EFMs with higher amounts of ALG particles degraded faster, which can probably be explained by the swelling and dissolution of ALG, which results in pores in the matrix. This resulted in increased contact between PLGA and the PBS medium, and thereby accelerateed the hydrolysis of the PLGA. The difference in the degradation rates between different EFMs became noticeable after 80 days of incubation, and after 105 days of incubation the EFMs disintegrated into small pieces.

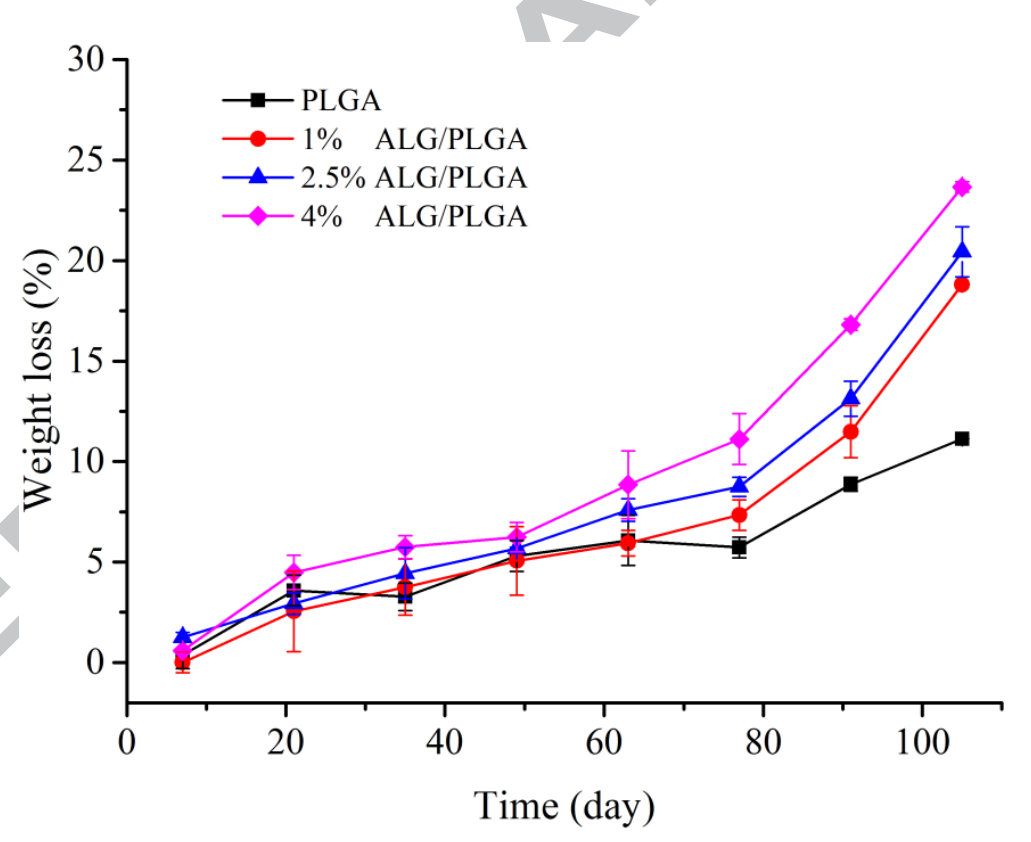

Fig. 6 Degradation of PLGA and PLGA/ALG (with 1, 2.5, 4 \% ALG) EFMs in PBS, pH 7.4 at $37^{\circ} \mathrm{C}$ for a period of 105 days. (mean $\pm \mathrm{SD}, \mathrm{n}=3$ ).

\subsection{Mechanical properties of the EFMs}

The mechanical properties of EFMs used as wound dressing are important as it is supposed to serve as a mechanical barrier or to provide physical support for cell growth and migration[34]. The result in Fig. 7 presents the typical stress-strain curves of the EFMs, where the Young's modulus of the EFMs describes stiffness (Fig. 8). Compared with the PLGA electrospun EFM, both the tensile 
strength and elongation rate of EFMs containing either ALG particles or CIP decreased dramatically. However, the Young's modulus and tensile strength of human skin are around 60-70 MPa and 17-21 MPa, respectively [39, 40]. Therefore, after blending with ALG particles or CIP, the tensile strength of EFM was much lower than that of human skin, but the Young's modulus was more close to human skin. During the wound healing period, the temporary protection at the injury site after epithelialization is only $15 \%$ tensile strength of the original skin, therefore it can be concluded that the electrospun CIP-ALG/PLGA EFMs are strong enough to protect the injury site.
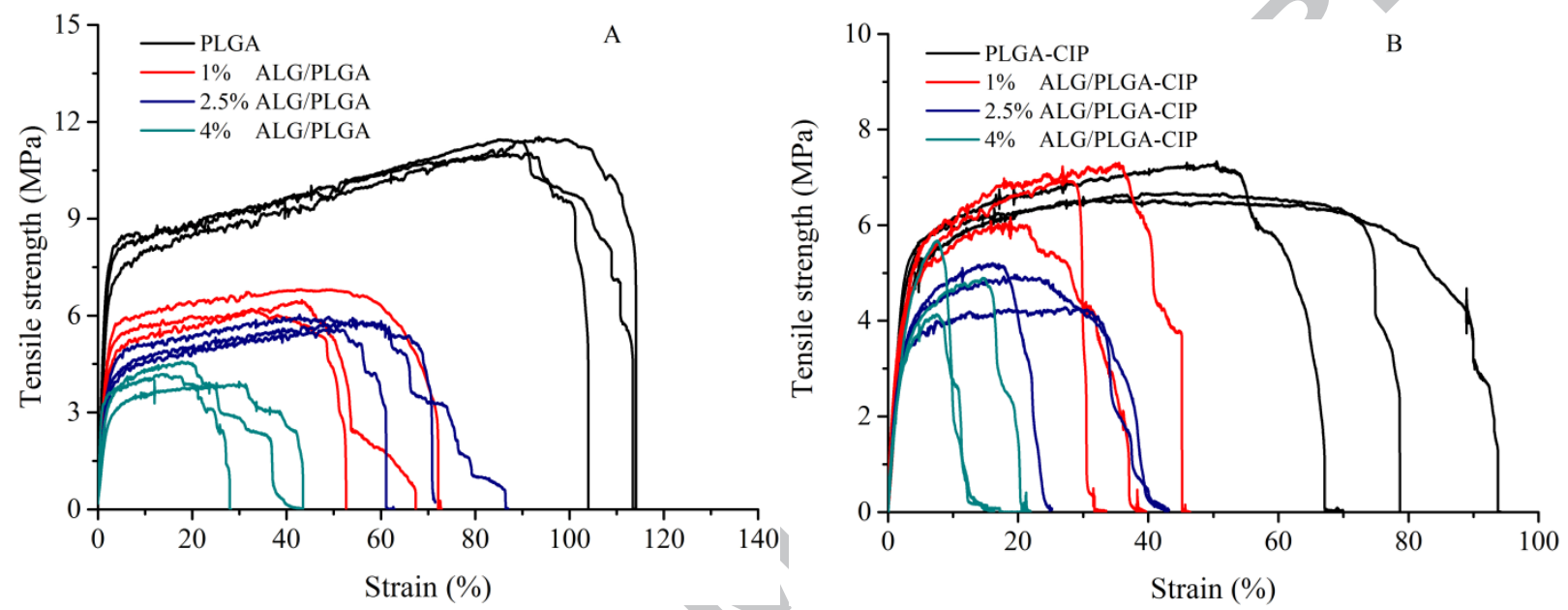

Fig. 7 Tensile strength of PLGA/ALG EFMs loaded/unloaded with CIP. A: PLGA EFM with/without ALG particles (1, 2.5 and $4 \%$ ); B: CIP-loaded PLGA EFM with/without ALG (1, 2.5 and $4 \%$ ) particles (three replicate measurements).

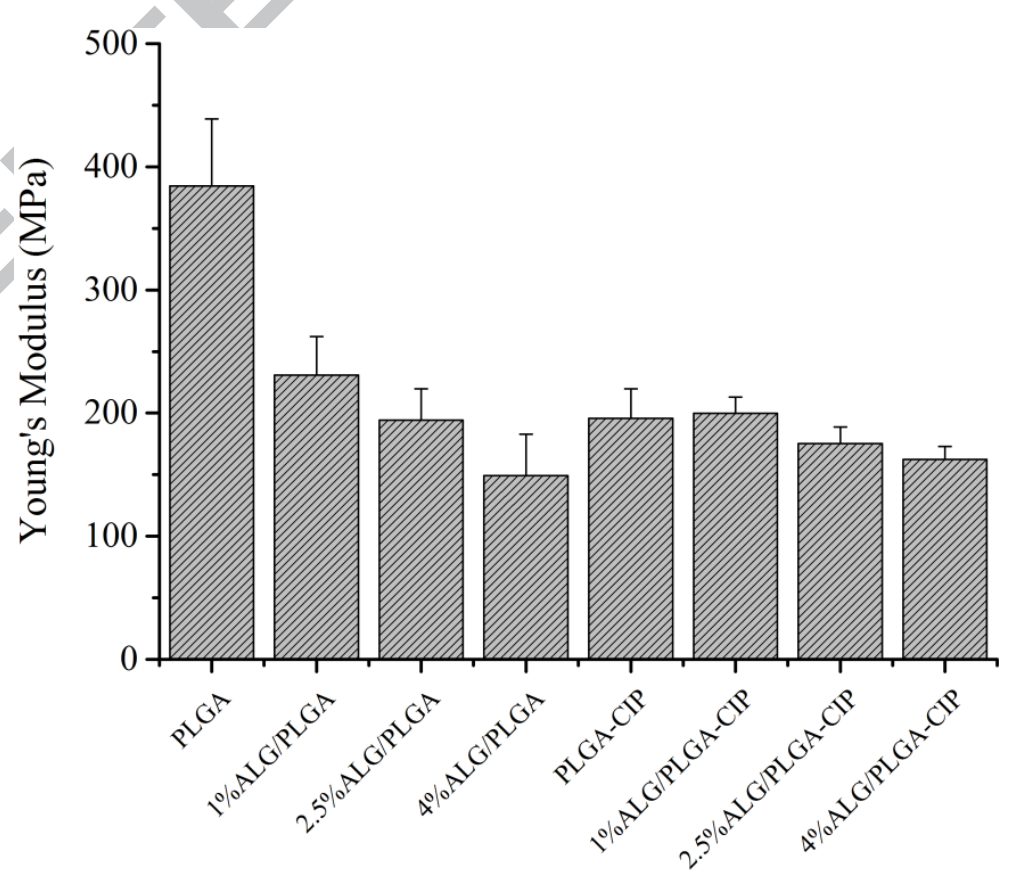

Fig.8 Young's modulus of the EFMs loaded/non-loaded with CIP (mean \pm SD, n=3). 


\subsection{Antimicrobial activity in solid culture medium}

The MIC for ciprofloxacin and $S$. aureus $15981 \mathrm{WT}$ was determined to be $0.125 \mu \mathrm{g} / \mathrm{mL}$ using the broth microdilution method, which was reached at the very beginning (within the first $15 \mathrm{~min}$ ) of the release study. The PLGA EFM did not show any antimicrobial activity, while all the CIP-loaded EFMs (drug loading, $1 \%$, w/w) exhibited an inhibition zone ranging from 35-45 mm (Fig. 10). All EFMs containing ALG showed an inhibition zone equal to or larger than PLGA-CIP EFM indicating that the antimicrobial activity was not compromised by the addition of the ALG particles. The EFM containing $4 \%$ ALG showed the best antimicrobial activity, and a significant improvement was found compared to CIP-PLGA EFM $(p=0.03)$. This can probably be explained by a higher amount of CIP released from the $4 \%$ ALG EFM compared to the other formulations within the studied time period of $24 \mathrm{~h}$. All the CIP-loaded EFMs showed good antibacterial effect, since the diameter of inhibition zone was larger than $21 \mathrm{~mm}[41]$.

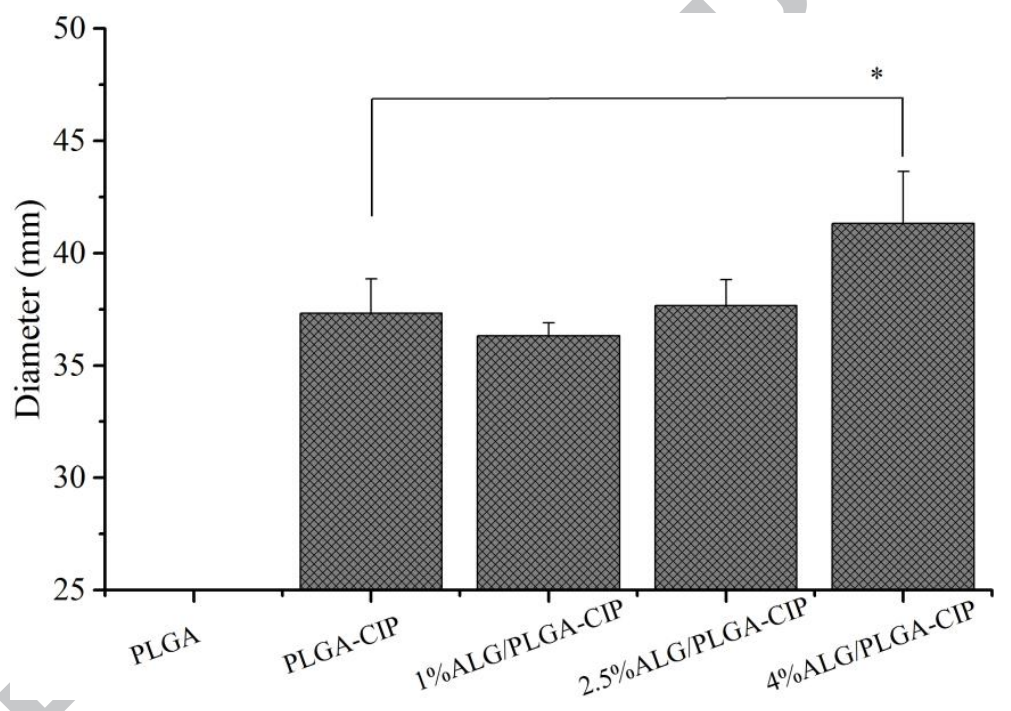

Fig. 10 The average diameter of the bacteria inhibition zone for CIP-loaded PLGA or ALG/PLGA EFMs within the time period of $24 \mathrm{~h}($ mean $\pm S D, n=3)$.

\section{Conclusion}

In this study, antibiotic-loaded electrospun hydrophobic polymer (PLGA) fibrous mats modified with hydrophilic polymer (soldium alginate) were prepared for wound healing purpose. The addition of hydrophilic polymer improved the wettability, water uptake potential of the hydrophobic mats and facilitated the release of the antibiotic at the early diffusion phase. Moreover, the resulting polymeric fibrous mats exhibited sufficient mechanical support for injure area. This study demonstrated the feasibility of incorporating a hydrophilic natural polymer into a hydrophobic synthetic polymer matrix by the electrospinning process to produce a promising wound dressing material.

\section{Acknowledgement}


This study was supported, in part, by Graduate School of Health and Medical Sciences of University of Copenhagen, Department of Pharmacy of University of Copenhagen, Department of Chemical Engineering, Biotechnology and Environmental Technology, University of Southern Denmark, the Danish Council for Independent Research, Technology and Production Sciences (FTP, Project 12-126515/0602-02670B), and University of Copenhagen Research Centre for Control of Antibiotic Resistance (UC-CARE). Furthermore, Line Hagner Nielsen would like to thank the Danish Research Council for Technology and Production (FTP), Project DFF-4004-00120B for financial support, and in addition, the Denmarks Grundforskningsfonds (project DNRF122) and Villum Fondens Center for Intelligent Drug Delivery and Sensing Using Microcontainers and Nanomechanics (IDUN) is acknowledged.

\section{Reference}

[1] S. Enoch, D.J. Leaper, Basic science of wound healing, Surgery (Oxford), 26 (2008) 31-37.

[2] G.C. Gurtner, S. Werner, Y. Barrandon, M.T. Longaker, Wound repair and regeneration, Nature, 453 (2008) 314-321.

[3] K. Moore, R. McCallion, R.J. Searle, M.C. Stacey, K.G. Harding, Prediction and monitoring the therapeutic response of chronic dermal wounds, Int Wound J, 3 (2006) 89-98.

[4] K. Cutting, R.J. White, Maceration of the skin and wound bed I: its nature and causes, J Wound care, 11 (2002) 275-278.

[5] K. Vowden, P. Vowden, Wound dressings: principles and practice, Surgery (Oxford), 32 (2014) 462-467.

[6] P.S. Murphy, G.R. Evans, Advances in wound healing: a review of current wound healing products, Plast Surg Int, 2012 (2012) 1-8.

[7] L.I. Moura, A.M. Dias, E. Carvalho, H.C. de Sousa, Recent advances on the development of wound dressings for diabetic foot ulcer treatment--a review, Acta Biomater, 9 (2013) 7093-7114.

[8] J.R. Davidson, Current concepts in wound management and wound healing products, Vet Clin North Am Small Anim Pract, 45 (2015) 537-564.

[9] S. Seaman, Dressing selection in chronic wound management, J Am Podiatr Med Assoc, 92 (2002) 24-33.

[10] C. McIntosh, Are hydrocolloid dressings suitable for diabetic foot ulcers?, Wounds Essentials (2007) 170-172.

[11] M.A. Fonder, G.S. Lazarus, D.A. Cowan, B. Aronson-Cook, A.R. Kohli, A.J. Mamelak, Treating the chronic wound: a practical approach to the care of nonhealing wounds and wound care dressings, J Am Acad Dermatol, 58 (2008) 185-206.

[12] J. Boateng, O. Catanzano, Advanced Therapeutic Dressings for Effective Wound Healing--A Review, J Pharm Sci, 104 (2015) 3653-3680.

[13] X. Zhu, W. Cui, X. Li, Y. Jin, Electrospun fibrous mats with high porosity as potential scaffolds for skin tissue engineering, Biomacromolecules, 9 (2008) 1795-1801.

[14] K.A. Rieger, N.P. Birch, J.D. Schiffman, Designing electrospun nanofiber mats to promote wound healing - a review, J Mater Chem B, 1 (2013) 4531-4541.

[15] W.J. Li, C.T. Laurencin, E.J. Caterson, R.S. Tuan, F.K. Ko, Electrospun nanofibrous structure: a novel scaffold for tissue engineering, J Biomed Mater Res, 60 (2002) 613-621.

[16] R. Jayakumar, M. Prabaharan, P.S. Kumar, S. Nair, H. Tamura, Biomaterials based on chitin and chitosan in wound dressing applications, Biotechnol Adv, 29 (2011) 322-337. 
[17] B.-M. Min, G. Lee, S.H. Kim, Y.S. Nam, T.S. Lee, W.H. Park, Electrospinning of silk fibroin nanofibers and its effect on the adhesion and spreading of normal human keratinocytes and fibroblasts in vitro, Biomaterials, 25 (2004) 1289-1297.

[18] K.S. Rho, L. Jeong, G. Lee, B.-M. Seo, Y.J. Park, S.-D. Hong, S. Roh, J.J. Cho, W.H. Park, B.M. Min, Electrospinning of collagen nanofibers: effects on the behavior of normal human keratinocytes and early-stage wound healing, Biomaterials, 27 (2006) 1452-1461.

[19] J.A. Matthews, G.E. Wnek, D.G. Simpson, G.L. Bowlin, Electrospinning of collagen nanofibers, Biomacromolecules, 3 (2002) 232-238.

[20] T. Freyman, I. Yannas, L. Gibson, Cellular materials as porous scaffolds for tissue engineering, Prog Mater Sci, 46 (2001) 273-282.

[21] D. Kai, S.S. Liow, X.J. Loh, Biodegradable polymers for electrospinning: towards biomedical applications, Mater Sci Eng C Mater Biol Appl, 45 (2014) 659-670.

[22] A.L.R. Pires, Â.M. Moraes, Improvement of the mechanical properties of chitosan-alginate wound dressings containing silver through the addition of a biocompatible silicone rubber, J Appl Polym Sci, 132 (2015), 41686 (1-9).

[23] N. Bhardwaj, S.C. Kundu, Electrospinning: a fascinating fiber fabrication technique, Biotechnol Adv, 28 (2010) 325-347.

[24] G.D. Mogosanu, A.M. Grumezescu, Natural and synthetic polymers for wounds and burns dressing, Int J Pharm, 463 (2014) 127-136.

[25] X. Liu, S.G. Baldursdottir, J. Aho, H. Qu, L.P. Christensen, J. Rantanen, M. Yang, Electrospinnability of Poly Lactic-co-glycolic Acid (PLGA): the Role of Solvent Type and Solvent Composition, Pharm Res, (2017) 1-12.

[26] M.A. de Moraes, A.C. Rodas, O.Z. Higa, M.M. Beppu, Membranes of biopolymer blends for wound healing applications. The 6th Latin American Congress of Artifical Organs and Biomaterials [27] S. Sahoo, S.L. Toh, J.C. Goh, A bFGF-releasing silk/PLGA-based biohybrid scaffold for ligament/tendon tissue engineering using mesenchymal progenitor cells, Biomaterials, 31 (2010) 2990-2998.

[28] F. Li, X.Y. Yin, X.Z. Yin, Instability of a leaky dielectric coaxial jet in both axial and radial electric fields, Phys Rev E Stat Nonlin Soft Matter Phys, 78 (2008) 036302 (1-11).

[29] X. Huang, C.S. Brazel, On the importance and mechanisms of burst release in matrixcontrolled drug delivery systems, J Control Release, 73 (2001) 121-136.

[30] X.Y. Sun, L.R. Nobles, H.G. Börner, R.J. Spontak, Field-Driven Surface Segregation of Biofunctional Species on Electrospun PMMA/PEO Microfibers, Macromol Rapid Commun, 29 (2008) 1455-1460.

[31] J. Gunn, M. Zhang, Polyblend nanofibers for biomedical applications: perspectives and challenges, Trends Biotechnol, 28 (2010) 189-197.

[32] M.V. Jose, V. Thomas, K.T. Johnson, D.R. Dean, E. Nyairo, Aligned PLGA/HA nanofibrous nanocomposite scaffolds for bone tissue engineering, Acta Biomater, 5 (2009) 305-315.

[33] L.R. Lakshman, K.T. Shalumon, S.V. Nair, R. Jayakumar, S.V. Nair, Preparation of Silver Nanoparticles Incorporated Electrospun Polyurethane Nano-fibrous Mat for Wound Dressing, J Macromol Sci, Part A, 47 (2010) 1012-1018.

[34] M. Prabaharan, R. Jayakumar, S. Nair, Biomedical applications of polymeric nanofibers[M], , Springer Science \& Business Media, 2012.

[35] D. Parikh, J. Edwards, B. Condon, A. Parikh, Silver-Carboxylate Ion-Paired Alginate and Carboxymethylated Cotton with Antimicrobial Activity, AATCC review, 8 (2008) 38-43.

[36] R. Fu, C. Li, C. Yu, H. Xie, S. Shi, Z. Li, Q. Wang, L. Lu, A novel electrospun membrane based on moxifloxacin hydrochloride/poly (vinyl alcohol)/sodium alginate for antibacterial wound dressings in practical application, Drug Deliv, (2014) 1-12. 
[37] A. Szentivanyi, T. Chakradeo, H. Zernetsch, B. Glasmacher, Electrospun cellular microenvironments: Understanding controlled release and scaffold structure, Adv Drug Deliv Rev, 63 (2011) 209-220.

[38] T.G. Park, Degradation of poly (D, L-lactic acid) microspheres: effect of molecular weight, J Control Release, 30 (1994) 161-173.

[39] A. Gallagher, A. Ní Annaidh, K. Bruyère, Dynamic tensile properties of human skin, in: IRCOBI Conference, 12-14 September 2012, Dublin (Ireland).

[40] M. Pawlaczyk, M. Lelonkiewicz, M. Wieczorowski, Age-dependent biomechanical properties of the skin, Postepy Dermatol Alergol, 30 (2013) 302-306.

[41] S.Q. Ali, A. Zehra, B.S. Naqvi, S. Shah, R. Bushra, Resistance pattern of ciprofloxacin against different pathogens, Oman Med J, 25 (2010) 294-298. 


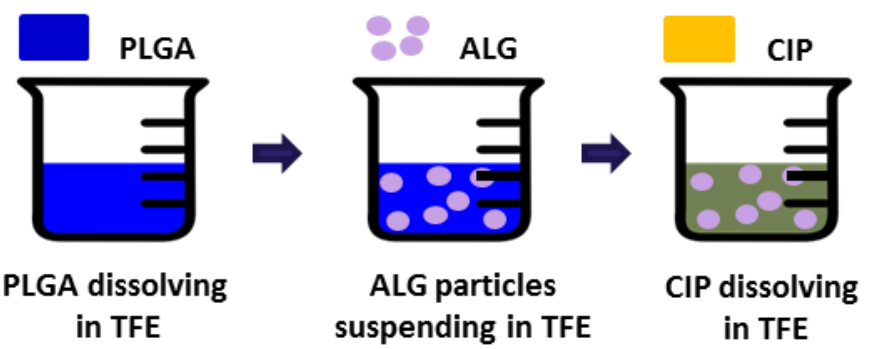

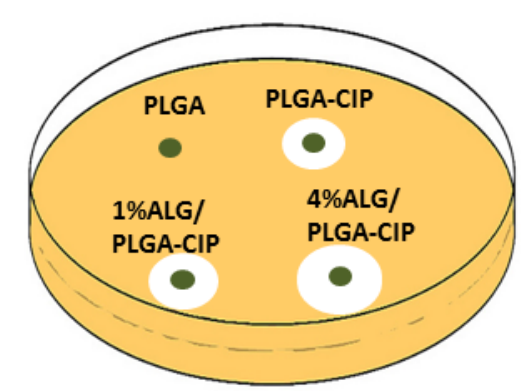

S. aureus inhibition zones of various electrospun fibrous mats

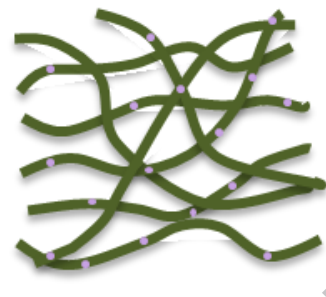

ALG/PLGA-CIP

fibrous mat

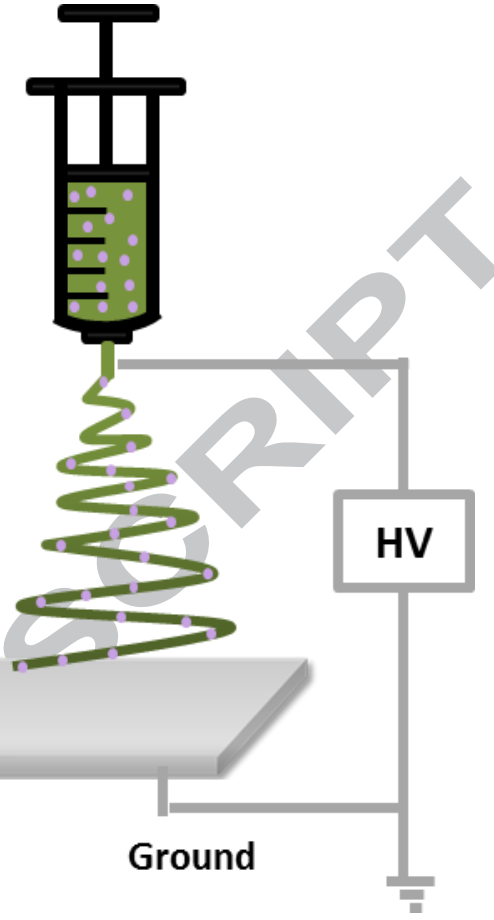

